\title{
Generation of four-qubit cluster of entangled coherent states in bimodal QED cavities
}

\author{
E. M. Becerra-Castro, W. B. Cardoso*, A. T. Avelar ${ }^{\dagger}$ and B. Baseia \\ Instituto de Física, Universidade Federal de Goiás, 74.001-970, Goiânia (GO), Brazil. \\ E-mail: *wesleybcardoso@gmail.com \\ E-mail: ${ }^{\dagger}$ avelardif.ufg.br
}

\begin{abstract}
A recent work [1] proposed a type of cluster entangled coherent states and its generation. Here we present an alternative experimental arrangement for its generation in bimodal QED cavities. The scheme employs a single two-level atom that interacts dispersively with cavity modes initially prepared in coherent states. The fidelity and success probability of the state preparation are obtained considering the influence of atomic velocity spread and atomic efficiency detection.
\end{abstract}

Entanglement is the most fantastic phenomenon of quantum mechanics and plays a fundamental role in quantum information theory and applications. There are various kinds of entangled states depending on the number of involved parties, e.g.: for two qubits there is only one example, the EPR state [2]; for three qubits there are two kinds of three-partite entangled states, the GHZ [3] and W [4] states; for four-qubit states there are at least nine kinds of entangled states, also named cluster states. In particular, in Refs. [5, 6] it was experimentally demonstrated that correlations in a four-qubit, linear cluster states given by

$$
\left|\phi_{4}\right\rangle=\frac{1}{2}(|0000\rangle+|0011\rangle+|1100\rangle-|1111\rangle)
$$

cannot be described in terms of the local realism. In fact, this state is not biseparable and has a genuine four-qubit entanglement [7]. Recently, Walther et al. [8] have implemented four-qubit cluster states encoded into the polarization state of four photons, which constituted an experimental proof of one-way quantum computing in Raussendorf and Briegel's "oneway" model [9] using initialized qubits in a highly entangled cluster state. In a recent paper Blythe and Varcoe [10], using the techniques of cluster state quantum computing, they showed how a scalable quantum computer could potentially be constructed using microwave cavity quantum electrodynamics (QED). These cluster state quantum computing uses a sequence of measurements on a lattice of entangled qubits in cluster states to perform quantum gate operations.

Based on potential applications of cluster states, schemes for their generation have been presented in various scenarios [1, 6, 8, 11, 12, 13, 14, 15, 16, 17]. In [11] Zou and Mathis it was proposed an unified scheme to generate GHZ states, W states, and cluster states of four distant atoms that are trapped separately in leaky cavities. They have also studied: a scheme to generate a four-photon polarization-entangled cluster state using only linear 
optical elements [12] and four-photon coincidence detection; and two additional schemes to generate the cluster states in the context of QED cavity [13]. Barrett and Kok [14] proposed a protocol to generate cluster states using spatially separated matter qubits and single-photon interference effects [15, 16]. In Ref.[17] the authors proposed methods of fidelity estimation and entanglement verification to experimentally produce four-qubit cluster states.

Recently, Munhoz et al. [1] investigated a cluster-type of four-qubit coherent states and its generation using five QED cavities, two Ramsey zones, a pair of two-level Rydberg atoms, an external classical field plus atomic ionization detectors. In view of the interesting idea and its potential applications, in this report we will present an alternative and simplified scheme for its generation in the same QED cavity context. We employ a pair of QED bimodal cavities, a single two-level (Rydberg) atom and a dispersive atom-field interaction. The simplicity of our scheme makes it attractive experimentally due to its feasibility in the present status of QED technology [18, 19]. This proposal requires the experimental setup shown in Fig. 1. The source $S$ ejects rubidium atoms which are velocity selected and prepared in the circular Rydberg state, one at a time, by appropriated laser beams in box the $B$. The relevant atomic levels $|g\rangle$ and $|e\rangle$, with the principal quantum numbers 50 and 51 , provide the atomic transition of $51.1 \mathrm{GHz} . R_{1}, R_{2}$ and $R_{3}$ stand for Ramsey zones which perform a resonant $\pi / 2$ pulse on the $e \rightarrow g$ transition. The two bimodal high-Q superconducting cavities $C_{1}$ and $C_{2}$ are Fabry-Perot resonators made of two spherical niobium mirrors with two orthogonally polarized $\mathrm{TEM}_{900}$ modes separated by $1.2 \mathrm{MHz}$ having the same Gaussian geometry (waist $w=6 \mathrm{~mm}$ ), and photon damping times of $130 \mathrm{~ms}$ [19]; $S$ stands for the microwave generator coupled to each cavity, $D_{e}$ and $D_{g}$ are atomic detectors. These cavities are prepared at low temperature $(T \simeq 0.6 K$ ) to reduce the average number of thermal photons; before starting the experiment the thermal field is erased [20].

To generate the cluster states in the four mode of the two bimodal cavities we need to make two operations: i) The first one occurs when the atom crosses the Ramsey zones $\mathrm{R}_{1}$, $\mathrm{R}_{2}$, and $\mathrm{R}_{3}$ and interacts with the classical fields resonant to the atomic transition between the states $|e\rangle$ and $|g\rangle$, with intensities adjusted to produce a $\pi / 2$ rotation in the atomic space, i.e.,

$$
\begin{aligned}
& |e\rangle \longrightarrow(|g\rangle+|e\rangle) / \sqrt{2} \\
& |g\rangle \longrightarrow(|g\rangle-|e\rangle) / \sqrt{2}
\end{aligned}
$$

ii) the second operation occurs in $C_{1}$ and $C_{2}$ and involves dispersive atom-field interactions with these cavity modes, one at a time, in such a way that the atom crossing the cavity in the excited state $|e\rangle(|g\rangle)$ produces a negative (positive) phase-shift in the desired mode state. The dispersive interaction is described by the effective atom-field Hamiltonian [18],

$$
H_{e f}=\hbar \frac{g_{\beta}^{2}}{\delta_{\beta}}\left[\left(\hat{a}_{\beta}^{\dagger} \hat{a}_{\beta}+1\right)|e\rangle\left\langle e\left|-\hat{a}_{\beta}^{\dagger} \hat{a}_{\beta}\right| g\right\rangle\langle g|\right],
$$

where $\hat{a}_{\beta}\left(\hat{a}_{\beta}^{\dagger}\right)$ is the boson annihilation (creation) operator for mode $\beta$, with $\beta=A, B$ for the cavity $C_{1}$; the same is valid for the cavity $C_{2}$ with $\beta=C, D ;|e\rangle\langle e|$ and $|g\rangle\langle g|$ are the atomic projectors, $g_{\beta}$ stands for the vacuum Rabi coupling with mode $\beta$, and $\delta_{\beta}$ is the detuning between atomic transition and the mode $\beta$. Although the cavity has two modes, the frequency splitting of $1.2 \mathrm{MHz}$ between them ensures the atom being efficiently coupled only to a single 


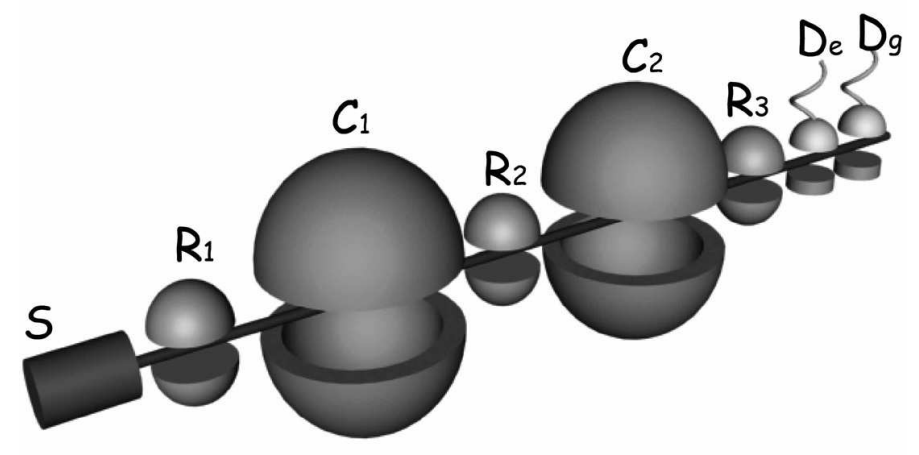

Figure 1. Schematic of the experimental setup for production of cluster states in bimodal QED cavities.

mode [19]. Then, using the Stark effect we choose the detuning with the mode $\beta$ which will interact in dispersive regime, i.e., $g_{\beta}^{2} \bar{n} / \delta_{\beta} \ll 1$, where $\bar{n}$ stands for the average photon number in the cavity. The evolution operator associated with Eq.(4) reads

$$
\hat{U}_{e f}=e^{-i \phi_{\beta}\left(\hat{a}_{\beta}^{\dagger} \hat{a}_{\beta}+1\right)}|e\rangle\left\langle e\left|+e^{i \phi_{\beta} \hat{a}_{\beta}^{\dagger} \hat{a}_{\beta}}\right| g\right\rangle\langle g|,
$$

where $\phi_{\beta}=g_{\beta}^{2} t_{\beta} / \delta_{\beta}$ and $t_{\beta}$ is the interaction time of the atom and the field in mode $\beta$.

Now we discuss our procedure to generate the cluster coherent state inside a two bimodal cavities. Initially, all the modes $A$ and $B$ of $C_{1}$ and $C$ and $D$ of $C_{2}$ are prepared in a coherent state $|i \alpha\rangle$ whereas the atom is prepared in the ground or excited states. The initial and final atomic states determine the type of cluster state obtained. Before entering in the first cavity the atom crosses a Ramsey zone $\mathrm{R}_{1}$ and the atomic state evolves to the superposition (2) and (3). Thus, the state of the whole system can be written as

$$
|\psi\rangle_{R_{1}}=\frac{1}{\sqrt{2}}(|g\rangle \pm|e\rangle)|i \alpha\rangle_{A}|i \alpha\rangle_{B}|i \alpha\rangle_{C}|i \alpha\rangle_{D},
$$

where the sign $+(-)$ comes from the atom initially prepared in the excited (ground) state. After that it enters the first cavity (detuning $\delta_{A}$ ) and interacts dispersively with the mode $A$ during a time $t_{A}$ to produce a phase shift $\phi_{A}=\pi / 2$. At the cavity axis, a Stark effect is applied to the atom tuning it dispersively $\left(\delta_{B}=\delta_{A}\right)$ with the mode $B$ for a time $t_{B}=t_{A}$. As result the state of the whole system becomes

$$
|\psi\rangle_{C_{1}}=\frac{1}{\sqrt{2}}\left(|g\rangle|-\alpha\rangle_{A}|-\alpha\rangle_{B}|i \alpha\rangle_{C}|i \alpha\rangle_{D} \mp|e\rangle|\alpha\rangle_{A}|\alpha\rangle_{B}|i \alpha\rangle_{C}|i \alpha\rangle_{D}\right) \text {. }
$$

When the atom emerges from the first cavity it crosses another Ramsey zone $\mathrm{R}_{2}$, tuned to the transitions (2) and (3). In this way we obtain

$$
\begin{aligned}
|\psi\rangle_{R_{2}} & =\frac{1}{2}\left(|g\rangle|-\alpha\rangle_{A}|-\alpha\rangle_{B}|i \alpha\rangle_{C}|i \alpha\rangle_{D}-|e\rangle|-\alpha\rangle_{A}|-\alpha\rangle_{B}|i \alpha\rangle_{C}|i \alpha\rangle_{D}\right. \\
& \left.\mp|g\rangle|\alpha\rangle_{A}|\alpha\rangle_{B}|i \alpha\rangle_{C}|i \alpha\rangle_{D} \mp|e\rangle|\alpha\rangle_{A}|\alpha\rangle_{B}|i \alpha\rangle_{C}|i \alpha\rangle_{D}\right) .
\end{aligned}
$$

Next the atom enters the cavity $C_{2}$ and interacts dispersively with the modes $C$ and $D$, in the same way as found in $C_{1}$. So the whole state results

$$
|\psi\rangle_{C_{2}}=\frac{1}{2}\left(|g\rangle|-\alpha\rangle_{A}|-\alpha\rangle_{B}|-\alpha\rangle_{C}|-\alpha\rangle_{D}+|e\rangle|-\alpha\rangle_{A}|-\alpha\rangle_{B}|\alpha\rangle_{C}|\alpha\rangle_{D}\right.
$$




$$
\left.\mp|g\rangle|\alpha\rangle_{A}|\alpha\rangle_{B}|-\alpha\rangle_{C}|-\alpha\rangle_{D} \pm|e\rangle|\alpha\rangle_{A}|\alpha\rangle_{B}|\alpha\rangle_{C}|\alpha\rangle_{D}\right) \text {. }
$$

Now, the atom crosses another Ramsey zone $\mathrm{R}_{3}$, leading the whole system to the state

$$
\begin{aligned}
|\psi\rangle_{R_{3}} & =\frac{1}{2 \sqrt{2}}\left[| g \rangle \left(|-\alpha\rangle_{A}|-\alpha\rangle_{B}|-\alpha\rangle_{C}|-\alpha\rangle_{D}+|-\alpha\rangle_{A}|-\alpha\rangle_{B}|\alpha\rangle_{C}|\alpha\rangle_{D}\right.\right. \\
& \left.\mp|\alpha\rangle_{A}|\alpha\rangle_{B}|-\alpha\rangle_{C}|-\alpha\rangle_{D} \pm|\alpha\rangle_{A}|\alpha\rangle_{B}|\alpha\rangle_{C}|\alpha\rangle_{D}\right) \\
& +|e\rangle\left(-|-\alpha\rangle_{A}|-\alpha\rangle_{B}|-\alpha\rangle_{C}|-\alpha\rangle_{D}+|-\alpha\rangle_{A}|-\alpha\rangle_{B}|\alpha\rangle_{C}|\alpha\rangle_{D}\right. \\
& \left.\left. \pm|\alpha\rangle_{A}|\alpha\rangle_{B}|-\alpha\rangle_{C}|-\alpha\rangle_{D} \pm|\alpha\rangle_{A}|\alpha\rangle_{B}|\alpha\rangle_{C}|\alpha\rangle_{D}\right)\right] .
\end{aligned}
$$

Finally the atomic detection projects the state of the two cavities in one of the following four-qubit coherent cluster states

$$
\begin{aligned}
\left|\chi_{1}^{(e, e)}\right\rangle & =\frac{1}{2}(|\alpha\rangle|\alpha\rangle|\alpha\rangle|\alpha\rangle+|\alpha\rangle|\alpha\rangle|-\alpha\rangle|-\alpha\rangle \\
& +|-\alpha\rangle|-\alpha\rangle|\alpha\rangle|\alpha\rangle-|-\alpha\rangle|-\alpha\rangle|-\alpha\rangle|-\alpha\rangle), \\
\left|\chi_{2}^{(g, g)}\right\rangle & =\frac{1}{2}(-|\alpha\rangle|\alpha\rangle|\alpha\rangle|\alpha\rangle+|\alpha\rangle|\alpha\rangle|-\alpha\rangle|-\alpha\rangle \\
& +|-\alpha\rangle|-\alpha\rangle|\alpha\rangle|\alpha\rangle+|-\alpha\rangle|-\alpha\rangle|-\alpha\rangle|-\alpha\rangle), \\
\left|\chi_{3}^{(g, e)}\right\rangle & =\frac{1}{2}(|\alpha\rangle|\alpha\rangle|\alpha\rangle|\alpha\rangle+|\alpha\rangle|\alpha\rangle|-\alpha\rangle|-\alpha\rangle \\
& -|-\alpha\rangle|-\alpha\rangle|\alpha\rangle|\alpha\rangle+|-\alpha\rangle|-\alpha\rangle|-\alpha\rangle|-\alpha\rangle), \\
\left|\chi_{4}^{(e, g)}\right\rangle & =\frac{1}{2}(|\alpha\rangle|\alpha\rangle|\alpha\rangle|\alpha\rangle-|\alpha\rangle|\alpha\rangle|-\alpha\rangle|-\alpha\rangle \\
& +|-\alpha\rangle|-\alpha\rangle|\alpha\rangle|\alpha\rangle+|-\alpha\rangle|-\alpha\rangle|-\alpha\rangle|-\alpha\rangle),
\end{aligned}
$$

where we have omitted the subscripts of the modes; the superscripts in Eqs.(11)-(14) indicate in which way the atomic state is prepared/detected. Note that the cluster states $\left|\chi_{1}^{(e, e)}\right\rangle$, $\left|\chi_{3}^{(g, g)}\right\rangle,\left|\chi_{3}^{(g, e)}\right\rangle$ and $\left|\chi_{4}^{(e, g)}\right\rangle$ correspond to $\left|C L U S T E R_{\alpha}^{+}\right\rangle,\left|C L U S T E R_{\alpha}^{-}\right\rangle,\left|C_{\alpha}^{+}\right\rangle$and $\left|C_{\alpha}^{-}\right\rangle$, respectively, found in [1]. In the same way, other types of cluster states appearing in [1] can be obtained by convenient choices of initial states. For example, from the initial state $|i \alpha\rangle_{A}|i \alpha\rangle_{B}|i \alpha\rangle_{C}|-i \alpha\rangle_{D}$ we obtain the cluster state $\left|L_{\alpha}^{ \pm}\right\rangle$given in [1].

Next, we calculate the total time $\tau$ spent for the generation of the four-qubit cluster coherent states. First, note that $\alpha$ must be chosen in a way that the cluster coherent states (11,14) be orthogonal and this choice determines the value of detuning $\delta$ satisfying the dispersive condition $\delta_{\beta} \gg g_{\beta} \sqrt{\bar{n}}$. Setting $\alpha=2$ leads to $\langle\alpha \mid-\alpha\rangle=e^{-2 \alpha^{2}} \simeq 10^{-3.5} \simeq 0$ and $\delta_{\beta}=40 g_{\beta}$. Now, using recent experimental data [19] for the central Rabi frequency $g_{\beta} \simeq 2 \pi \times 51 \mathrm{kHz}\left(\right.$ with $\beta=A, B$ ) one obtains the interaction time $t_{\beta}=\pi \delta_{\beta} / 2 g_{\beta}^{2}=196 \mu \mathrm{s}$ to produce a phase shift $\pi / 2$ in the state of mode $\beta$. This requires the atomic velocity $v=\sqrt{\pi} w /\left(t_{A}+t_{B}\right)=27 \mathrm{~m} / \mathrm{s}$ to get an effective atom-field interaction time when taking into account the variation of the Rabi frequency due to the atomic motion across the Gaussian cavity mode. The selected atomic velocity belongs to the typical interval available in laboratories $20-500 \mathrm{~m} / \mathrm{s}$. The total time required for the interaction of the atom with the four modes in the two cavities is $\tau=0.784 \mathrm{~ms}$. The scheme presented here can be mathematically 


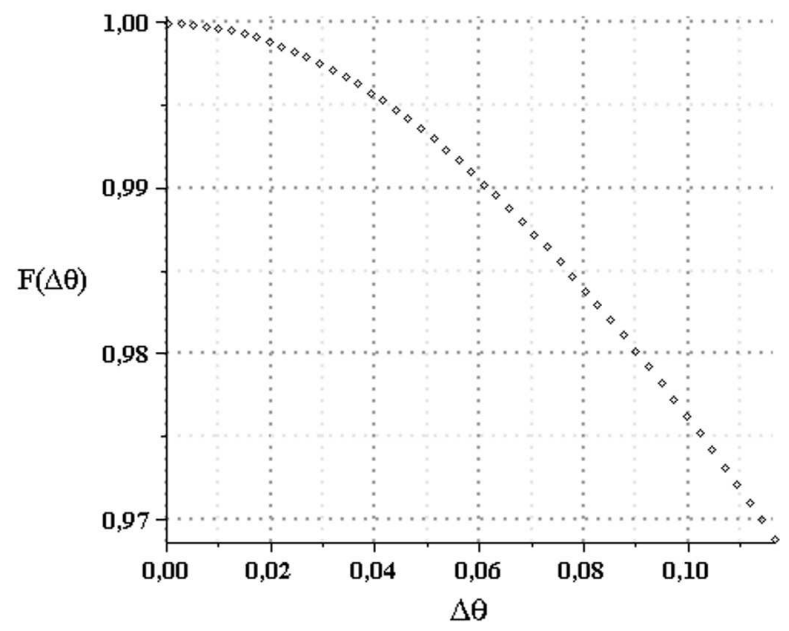

Figure 2. Fidelity of the prepared cluster state comparing the ideal and real cases, obtained with the influence of the velocity spread included.

generalized for hight orders of particles in cluster coherent state simply increasing more cavities in the experimental apparatus. However one should take into account the total time to accomplish the procedure and like this to count the decoherence effects in the field state in the cavity, that in those cases can be important.

Till now we have considered all calculations in an ideal experiment. In a realistic case the effect of velocity spread, detection efficiency, and dissipation should be taken into account. First, we know that the atomic velocity determines the pulse $\theta$ in the Ramsey zones and the phase shift $\phi$ in the cavity mode, so any atomic velocity spread produces uncertainties in the values of $\theta$ and $\phi$. We calculate the fidelity comparing the ideal cluster and real cluster states, obtained with the influence of the velocity spread included. For experimental velocity spreads $\Delta v= \pm 2 \mathrm{~m} / \mathrm{s}$ [18] the fidelity is $\simeq 97 \%$ as showed in Fig.(2); second, there is no problem with respect to detector efficiency since the scheme works with a single atom; third, the total time $\tau$ of experiment ( $\tau=0.784 \mathrm{~ms}$, neglecting the spatial separation between cavities) is much lesser than photon damping times $\left(t_{d}=130 \mathrm{~ms}\right)$ and atomic radiative times $\left(T_{a} \simeq 30 \mathrm{~ms}\right.$, for Rydberg atoms); so, we will neglect dissipation and the scheme may be experimentally feasible in the realm of microwave.

In summary, inspired on a previous work [1] we have presented an alternative scheme to generate a four-qubit cluster of coherent states based on the potential applications for quantum computing [9, 8, 10].Cavity QED is widely believed to be an excellent system for quantum gate operations [10]. The our method to generate the cluster coherent state is suitable for applications in the scheme for cluster-state quantum computing of the Ref. [10]. It employs two bimodal QED cavities and a single two-level Rydberg atom, constituting an economic version compared with [1]. Here we have considered the variation of the Rabi frequency due to the atomic motion across the Gaussian cavity mode: it results in the total time of experiment $\tau=0.784 \mathrm{~ms}$, smaller than that in [1]. In addition, the success probability to get a specific cluster state of the family in Eqs.(11)-(14) is 50\%, instead of the $25 \%$ found in [1]. Finally, 
we have taken advantage of a recent result by the Haroche's group [19], allowing us to neglect the decoherence of the state during the generation process.

\section{Acknowledgments}

We thank the CAPES, CNPq, FUNAPE, Brazilian agencies, and PRPPG/UFG, for the partial supports.

\section{References}

[1] Munhoz P P, Semião F L, Vidiella-Barranco A and Roversi J A 2007 arXiv:0705.1549v1 [quant-ph]

[2] Lo H K and Popescu S 2001 Phys. Rev. A 63022301

[3] Dur W, Vidal G and Cirac J I 2000 Phys. Rev. A 62062314

[4] Acin A, Bru $\beta$ D, Lewenstein M and Sanpera A 2001 Phys. Rev. Lett. 87040401

[5] Walther P, Aspelmeyer M, Resch K J and Zeilinger A 2005 Phys. Rev. Lett. 95020403

[6] Kiesel N, Schmid C, Weber U, Tóth G, Gühne O, Ursin R and Weinfurter H 2005 Phys. Rev. Lett. 95 210502

[7] Tokunaga Y, Yamamoto T, Koashi M and Imoto N 2006 Phys. Rev. A 74 020301(R)

[8] Walther P, Resch K J, Rudolph T, Schenck S, Weinfurter H, Vedral V, Aspelmeyer M and Zeilinger A 2005 Nature 434169

[9] Raussendorf R and Briegel H J 2001 Phys. Rev. Lett. 865188

[10] Blythe P J and Varcoe B T H 2006 New J. Phys. 8231

[11] Zou X B, Pahlke K and Mathis W 2004 Phys. Rev. A 69052314

[12] Zou X B and Mathis W 2005 Phys. Rev. A 72013809

[13] Zou X B and Mathis W 2005 Phys. Rev. A 71032308

[14] Barrett S D and Kok P 2005 Phys. Rev. A 71 060310(R)

[15] Borhani M and Loss D 2005 Phys. Rev. A 71034308

[16] Cho J and Lee H W 2005 Phys. Rev. Lett. 95160501

[17] Tokunaga Y, Yamamoto T, Koashi M and Imoto N 2005 Phys. Rev. A 71 030301(R)

[18] Raimond J M, Brune M and Haroche S 2001 Rev. Mod. Phys. 73565

[19] Gleyzes S, Kuhr S, Guerlin C, Bernu J, Deléglise S, Hoff U B, Brune M, Raimond J M and Haroche S 2007 Nature 446297.

[20] Nogues G, Rauschenbeutel A, Osnaghi S, Brune M, Raimond J M and Haroche S 1999 Nature 400239 